\title{
Are There Vaccines Against Violence?
}

\author{
Joao Paulo Martins do Carmo* \\ Sao Paulo State University, Brazil \\ *Corresponding author: Joao Paulo Martins do Carmo, Sao Paulo State University, Campus Itumbiara-GO, Brazil
}

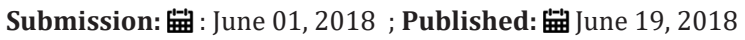

\section{Editorial}

Considering the transdisciplinar character of this Cohesive Journal of Microbiology and Infectious Disease, I would like to approach the violence and security question from the point of view of the Public Health, comparing them, shortly, to the cancer development (carcinogenesis), once cancer and violence are both Public Health problems. I also would like to approach it according to the holistic and ecologic theory of Fritj of Capra from his seminal masterpieces "The Web of Life" and "Turning Point".

To begin with, the carcinogens (compounds that cause cancer) may be biological (for instance, mainly of bacterial origin, for Helycobacter coli causing stomach cancer and other types; or viral origin, for which some examples are lymphomas and nasopharynx carcinoma caused by Epstein Barr; or lymphoma by Human T Lymphotropic Virus - HTLV; Human Papilloma Virus - HPV causing cervix or oral cancer; and hepatocarcinomas caused by $\mathrm{B}$ or $\mathrm{C}$ hepatitis virus-HBV/HCV), chemical (drugs, toxins, venoms, hormones, poluents, occupational etc) or physical (radiation being the most important representative of this group).

According to the Ecological and Complexity Theories from Capra [1], best explained in his book "The Web of Life," all living beings are connected to something bigger (the web of life, as an invisible thread). Let us focus here on the human beings. Thus, consider that an atom of any chemical element is constituted by patterns maintained in the micro and macroscopic levels, that, as we may see further, may have micro and macrocosmic implications. One can observe empirically the draws of any chemical element of the periodic table in a chemistry book, composed by protons with positive charges and electrons with negative charges spinning around them. Moreover, the number of protons are specific for each atom and they are denser than the electrons, thus, with lesser mobility. One may think that protons and electrons are kept together in an atom by the differences in charges, but they are stable according to some rules, the same way that the moon spins around the earth by the gravity law. To sum up, the most known rule is the Octet one, which establishes that atoms are stable with 2 or 8 electrons in their last layer. If they do not have this amount of electrons, they tend to search for stability acquiring/giving electrons in/to other atoms to complete their last layer. This is the chemical and physical basis to form new chemicals, such as ionic compounds or covalent molecules, through the chemical reactions. Some reactions may leave compounds with free electrons, making them very reactive. These reactive species are called free radicals. Compounds very reactive, such as oxygen and nitrogen species are normally avoided or eliminated by natural antioxidants found in diet or produced naturally in our bodies. The excess of free radicals, as they are oxidants, may lead to cellular senescence, and finally, to cancer development, once they may induce breaks in the DNA strand (s) and/or mutation.

In the multicellular beings, molecules and macromolecules are grouped to form organelles; organelles work together to perform the function of an specific cell; cells are united to form tissues that exert basically the same function; different tissues with different functions are grouped to form the organs; organs shape systems and systems collaborate in coordination to maintain the homeostatic survival of an individual (that can be regarded as a cell, as we will see further). Also according to Ecology, individuals from the same species make a population, which in conjunction with beings from other species (a house with cats, dogs, birds and plants, for instance) make up a community. Add abiotic factors (temperature, light, radiation, $\mathrm{pH}$, water etc), or in other words, environmental factors, to the community and we have an ecosystem. The many ecosystems in Earth form the biosphere, which is where living beings are in the Earth globe.

Following the Gaia Theory Capra [1], we can consider the Earth as a huge living being, and thus subject to the physiological regulatory laws to keep the homeostasis of an individual living organism, whether it is unicellular or multicellular. But this is subject to another editorial. Depending on the instrument we use to make our observation (magnifying glass, microscope or telescope), we can analyze cells, individuals, communities, ecosystems or galaxies. Only now, from this point of view, it becomes easier to understand that there are some natural patterns that we do not fully understand, but it is intriguing to think that the pattern of organization of an atom is similar to the pattern of organization of the solar system, with the Sun at the center (heliocentric theory), and planets orbiting around them, as if they were electrons spinning around their atom. If we think of the milky way as an example, we 
know today that there are other galaxies, so why not hypothesize other life forms in other planets? Scientists claim to have found bacterias in high temperatures of Siberian volcano's, suggesting that if in hospitable spheres of Earth can allow their survival, why not in other planets with similar atmosphere?

Without going into the details of carcinogenesis mechanisms, which should already be addressed extensively in specific journals, we can classify these mechanisms that lead to cancer progression between two types of gene expression related to the cell cycle: protooncogenes and tumor suppressor genes. The former are related to direct control of cell proliferation, while the latter act through their products to prevent a defective cell from progressing, causing its death by apoptosis. When a mutation occurs in one of these genes, there is a lack of control of the cell cycle, in the first case, allowing uncontrolled proliferation; and in the second case, if a cell does not enter apoptosis, its prolonged survival allows new mutations to occur, and may gain characteristics of a proliferative cell without homeostatic control. It is at this point that the concepts concerning the issue of public security, violence and cancer converge. If we consider each individual as a cell, public security may be compared to the checkpoints of mitosis in normal and cancerous cells. Let us think of a particular type of cell, the lymphocytes, and possible types of cancer that affect them: leukemias and lymphomas.

The lymphocytes rise from precursors in the bone marrow and undergo differentiation (selection of tools for a professional repertoire, as the children who go to a technical school to learn a specific job) and maturation (when they decide to go to college to specialize in a biological or engineer area), for example. Focusing on T cells (similar patterns occurs in B cells with specific details), there are some mechanisms that occur in the thymus, a central lymphoid organ of the immune system, to allow the cells to migrate (positive selection) or not (negative selection) to the periphery (peripheral lymphoid organs, like the tonsils or lymphnodes) to exert their effector function. These processes are called "Thymic Education" Abbas [2], and errors in this process, such as a cell that should be deleted but is not, can lead to the development of autoimmune diseases, once it will look for auto antigens in the periphery (it reminds the massacres in high schools around the world). It is not known the exact etiology of the autoimmune diseases, but for some of them, viral or bacterial infections may be involved in multiple sclerosis or bacterial endocarditis, respectively. In this latter case, for instance, the protein of the cardiac muscle fiber may be confused by the immune system as a streptococcal protein, causing muscle cell recognition as a foreign antigen and leading to its destruction by auto reactive $\mathrm{T}$ cells. In other situations, errors in mitosis checkpoints early in differentiation, such as in the stem cell stage, may lead to acute and chronic lymphoid and myeloid leukemias and also lymphomas. Briefly, acute leukemia occurs when there are altered precursors and chronic one when defects occur in mature cells, such as neutrophils, monocytes or lymphocytes.

Thus, in a general and rather simplified way, I want to hypothesize with this theory that in the same way that a lymphocyte needs to be monitored throughout its life (by mechanisms called immune vigilance, among others, that works to avoid the development of cancer and is similar to the tolerance mechanisms that occur in thymus to prevent autoimmune diseases), a CD8+ T lymphocyte in turn does exactly this function: it looks for defective antigens on the surface of all cells and if there is something weird, it has the role of communicating other cells through the production of cytokines (letters of advertence); however, as a police officer, if something goes wrong (such as a drug addict that disobeys him, representing a T lymphocyte finding a cancer cell or infected with a virus), it is licensed to kill the defective cell, in a process known as "the kiss of death". In this case, we must remember that the function of the immune system, unlike what most people think or learn or is taught in schools and colleges, is not the warlike vision of destroying potential foreign pathogens (for thus we would have to destroy our micro biota, and that does not happen all the time). The main function of the immune system is the homeostasis or immune surveillance, maintaining the integrity of the organs, cells and the life of individuals, destroying pathogens only when they offer danger, and preferably without causing tissue injury Matzinger [3] reviewed by Pradeau \& Cooper [4]; Kono \& Rock [5]. That's not the case most of the time either.

Thus, in an analogous way, we think that, in our current society, violence persists in some countries where we did not know as public policies to provide adequate education and employment for the various component individuals, causing them to deviate from professional maturation and seek deviations or shortcuts, such as a cancer that evades the immune response by producing immunosuppressive molecules and by deceiving effector $\mathrm{T}$ lymphocytes inducing them to become regulatory $\mathrm{T}$ lymphocytes (Tregs). In this way, the cancer corrupts the immunovigilant cells, and in some situations, it is necessary to request reinforcement, either in the form of vaccines, as prevention (HPV, HBV, etc) or immunotherapy (with monoclonal antibodies) for already established cancers. However, if there is metastasis, when the cancer is already spread throughout the body, there is even smaller chance of reestablishment of homeostasis and individual integrity. When this happens, the society as a whole becomes a victim, a hostage of violence; and violence, as the infectious diseases, is contagious.

A cancer that is diagnosed earlier has higher chances of being cured and eradicated. It will be even better if it can be prevented, as it does for the cervix cancer caused by HPV or hepatocarcinoma caused by HBV or HCV. Thus, the possibility to avoid violence rises with the improvement in education and not with the ostensive vigilancy of the police officers. Violence creates more violence and in the same way, kindness generates more kindness. In England, there is an effort of the National Health Service (NHS) to increase awareness and strengthen the commitment to prevention among government, NHS, local authorities, private and volunteer sectors, as well as education, employers and other agencies, in the common sense of divulgate the latest evidences that show that the main risk factors that make individuals, families and communities vulnerable to violence are changeable and thus, preventable Bellis [6]. As a byproduct, increasing the number of cameras ("immunovigilance") in London public areas has helped to decrease violent episodes, including robbery Dahlberg Ll \& Krug Eg [7]. 
Thus, it is our view that valuing Elementary Education might be a promising vaccine against future violence, because some works show that someone who has been exposed to the violence, specially as a child, tends to be involved in violence episodes again later in life Bellis [6].

\section{References}

1. Capra F (1997) The web of life: A new scientific understanding of living systems.

2. Abbas (2015) Cellular and molecular immunology. Elsevier, USA.

3. Gallucci S, Matzinger P (2001) Danger signals: SOS to the immune system. Curr Opin Immunol 13(1): 114-149.
4. Pradeau T , Cooper EL (2012) The danger theory: 20 years later. Front Immunol 3: 287.

5. Kono H, Rock L (2008) How dying cells alert the immune system to danger. Nat Rev Immunol 8(4): 279-289.

6. Bellis Ma, Hughes K, Perkins C, Bennett A (2012) Protecting people, Promoting health: A Public Health approach to violence prevention for England. National Health Services, UK, pp. 1-69.

7. Dahlberg Ll, Krug Eg (2002) Violence: a global public health problem. In: Krug E, Dahlberg LL, Mercy JA, Zwi AB, Lozano R, (Eds.), World Report on Violence and Health, World Health Organization, Switzerland, pp. 1-56.
Creative Commons Attribution 4.0

International License

For possible submissions Click Here
Submit Article

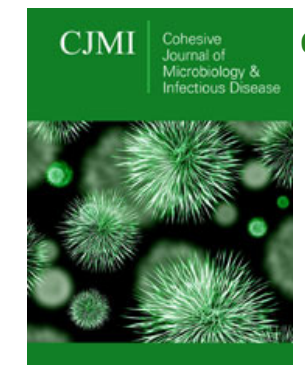

Cohesive Journal of Microbiology \& Infectious Disease

\section{Benefits of Publishing with us}

- High-level peer review and editorial services

- Freely accessible online immediately upon publication

- Authors retain the copyright to their work

- Licensing it under a Creative Commons license

- Visibility through different online platforms 